05

\title{
Невзаимное рассеяние нейтронов геликоидальными ферромагнетиками
}

\author{
(ㄱ Д.А. Татарский, А.А. Фраерман \\ Институт фозиики микроструктур РАН, \\ Нижний Новгород, Россия \\ E-mail: tatarsky@ipmras.ru
}

\begin{abstract}
Приведены расчеты рассеяния неполяризованных нейтронов на кристаллах с геликоидальным магнитным упорядочением. Показано, что положение пиков малоугловой дифракции нейтронов зависит от величины и направления внешнего магнитного поля, приложенного вдоль оси геликоида.
\end{abstract}

Работа выполнена при поддержке грантов РФФИ.

\section{1. Введение}

В настоящее время активно исследуются магнитные свойства нецентросимметричных кубических магнетиков. К ним относятся соединения $\mathrm{Si}$ и $\mathrm{Ge}$ с переходными металлами $\mathrm{Mn}, \mathrm{Fe}, \mathrm{Co}$ и $\mathrm{Ni}[1,2]$. Из-за антисимметричного обменного взаимодействия Дзялошинского-Мория [3,4] в этих кристаллах реализуется геликоидальное распределение намагниченности, причем период магнитной сверхструктуры существенно превышает межатомное расстояние. Дополнительное слабое анизотропное взаимодействие фиксирует направление геликоида вдоль одного из особых кубических направлений [5]. При приложении внешнего магнитного поля вдоль оси геликоида распределение магнитной индукции становится некомпланарным. Согласно теоретическим предсказаниям [6-8] и эксперименту [9], рассеяние нейтронов на системах с некомпланарным распределением магнитного поля невзаимно. В настоящей работе мы рассчитываем величину эффектов, обусловленных невзаимным рассеянием нейтронов при их малоугловой дифракции на кристаллах $\mathrm{MnSi}$ с геликоидальным распределением намагниченности.

\section{2. Расчет малоуглового рассеяния нейтронов}

Уравнение Шредингера для движения нейтронов в среде с геликоидальным распределением магнитного поля в безразмерных единицах $\left(2 m V_{0} / \hbar=1\right)$ имеет вид

$$
-\Delta \hat{\psi}+\hat{\psi}+k_{B}^{2}\left(\begin{array}{cc}
\cos \theta & \sin \theta e^{-i q z} \\
\sin \theta e^{i q z} & -\cos \theta
\end{array}\right) \hat{\psi}=k_{0}^{2} \hat{\psi}
$$

где $m$ - масса нейтрона, $V_{0}-$ величина ядерного потенциала, $\hat{\psi}-$ спинорная волновая функция нейтрона, $k_{B}^{2}=\frac{\mu_{n} B}{V_{0}}-$ величина взаимодействия магнитного момента нейтрона $\mu_{n}$ с магнитным полем $B$ в безразмерных единицах, $\theta$ - половина угла раствора конуса геликоида, $k_{0}^{2}$ - энергия падающего пучка в безразмерных единицах, $q$ - обратный вектор геликоида. Спектр и волновые функции нейтронов, описываемых уравнением (1), хорошо известны $[10,11]$ и могут быть записаны следующим образом:

$$
\hat{\psi}_{ \pm}(k, p)=\left(\begin{array}{c}
\frac{\delta_{ \pm}(k)}{\sqrt{1+\delta_{ \pm}^{2}(k)}} e^{i q z / 2} \\
e^{i q z / 2}
\end{array}\right) e^{i k z} e^{i p x}
$$

где

$$
\delta_{ \pm}(k)=\frac{k_{B}^{2} \cos \theta-k q \pm \sqrt{k_{B}^{4}-2 k q k_{B}^{2} \cos \theta+k^{2} q^{2}}}{k_{B}^{2} \sin \theta} .
$$

При этом энергия падающего пучка $k_{0}^{2}$ и проекции $k, p$ волнового вектора в среде связаны дисперсионным соотношением

$$
k_{0}^{2}=k^{2}+p^{2}+\frac{q^{2}}{4}+1 \pm \sqrt{k_{B}^{4}-2 k q k_{B}^{2} \cos \theta+k^{2} q^{2}} .
$$

Рассмотрим прохождение и отражение нейтронов от среды конечной толщины L c коническим распределением намагниченности (рис. 1). В соответствии с (1) ось $z$

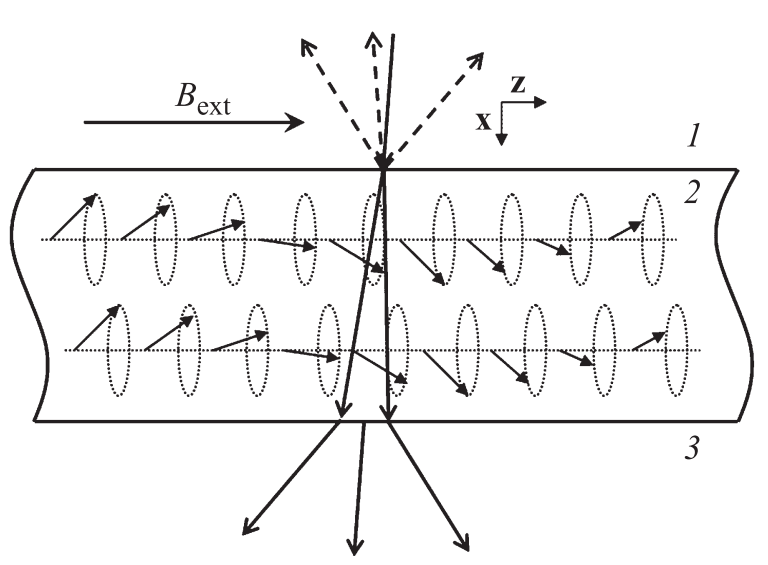

Рис. 1. Схема малоуглового рассеяния нейтронов на монокристалле с геликоидальным распределением намагниченности во внешнем магнитном поле. Помимо зеркального и прошедшего пучков также есть по два пучка, отвечающих дифракции на периоде геликоида при прохождении и отражении. Цифры 1-3 отвечают областям с соответствующим значением ядерного потенциала. 
направим вдоль оси геликоида, а ось $x$ - так, чтобы плоскость $x z$ совпадала с плоскостью рассеяния. Для нахождения коэффициентов отражения и прохождения необходимо найти волновые функции, удовлетворяющие условиям непрерывности на границах $1-2$ и $2-3$

$$
\left\{\begin{array}{l}
\hat{\psi}_{0}(x=0)+\hat{\psi}_{R}(x=0)=\hat{\psi}_{H}(x=0), \\
\partial_{x} \hat{\psi}_{0}(x=0)+\partial_{x} \hat{\psi}_{R}(x=0)=\partial_{x} \hat{\psi}_{H}(x=0), \\
\hat{\psi}_{H}(x=L)=\hat{\psi}_{T}(x=L), \\
\partial_{x} \hat{\psi}_{H}(x=L)=\partial_{x} \hat{\psi}_{T}(x=L),
\end{array}\right.
$$

где $\hat{\psi}_{0, R, T}-$ соответствующие волновые функции падающих, отраженных и прошедших нейтронов, $\hat{\psi}_{H}-$ волновая функция нейтронов в среде, $\partial_{x}-$ производная по $x$. Поскольку граничные условия должны выполняться в любой точке $z$ при $x=0, L$ и все волновые функции имеют осциллирующий характер, пространственные частоты по оси $z$ в левых и правых частях равенств (4) должны совпадать. Считая, что $\hat{\psi}_{0}\left(k_{0 z}, p_{0}\right)=\left(\begin{array}{l}i_{1} \\ i_{2}\end{array}\right) \exp \left[i k_{0 z} z\right] \exp \left(i p_{0} x\right)$ и учитывая вид волновых функций (2) в среде, легко находим, что искомые волновые функции $\hat{\psi}_{H, R, T}$ должны быть записаны в следующем виде:

$$
\begin{aligned}
& \hat{\psi}_{R}=\left(\begin{array}{c}
r_{11} \\
r_{22}
\end{array}\right) \exp \left[i k_{0 z}\right] \exp \left[-i p_{0 x} x\right]+\left(\begin{array}{c}
r_{21} \\
0
\end{array}\right) \exp \left[i\left(k_{0 z}-q\right) z\right] \\
& \quad \times \exp \left[-i p_{-} x\right]+\left(\begin{array}{c}
0 \\
r_{12}
\end{array}\right) \exp \left[i\left(k_{0 z}+q\right) z\right] \exp \left[-i p_{+} x\right], \\
& \hat{\psi}_{H}=C_{1} \hat{\psi}_{+}\left(k_{0 z}+\frac{q}{2}, p_{1}\right)+C_{2} \hat{\psi}_{-}\left(k_{0 z}+\frac{q}{2}, p_{2}\right) \\
&+C_{3} \hat{\psi}_{+}\left(k_{0 z}-\frac{q}{2}, p_{3}\right)+C_{4} \hat{\psi}_{+}\left(k_{0 z}-\frac{q}{2}, p_{4}\right) \\
&+C_{5} \hat{\psi}_{+}\left(k_{0 z}+\frac{q}{2},-p_{1}\right)+C_{6} \hat{\psi}_{-}\left(k_{0 z}+\frac{q}{2},-p_{2}\right) \\
&+C_{7} \hat{\psi}_{+}\left(k_{0 z}-\frac{q}{2},-p_{3}\right)+C_{8} \hat{\psi}_{-}\left(k_{0 z}-\frac{q}{2},-p_{4}\right), \\
& \hat{\psi}_{T}=\left(\begin{array}{l}
t_{11} \\
t_{22}
\end{array}\right) \exp \left[i k_{0 z}(z-L)\right] \exp \left[-i p_{0 x} x\right]+\left(\begin{array}{c}
t_{21} \\
0
\end{array}\right) \\
& \times \exp \left[i\left(k_{0 z}-q\right)(z-L)\right] \exp \left[-i p_{-} x\right]+\left(\begin{array}{c}
0 \\
t_{12}
\end{array}\right) \\
& \times \exp \left[i\left(k_{0 z}+q\right)(z-L)\right] \exp \left[-i p_{+} x\right],
\end{aligned}
$$

где $r_{11,22}$ - амплитуды волновой функции, соответствующие зеркальному отражению, $r_{12,21}$ - амплитуды дифракционных пиков в отраженном пучке, $t_{11,22}-$ амплитуды волновой функции, соответствующие прямому прошедшему пучку, $t_{12,21}$ - амплитуды дифракционных пиков первого порядка в прошедшем пучке и $\left(k_{0 z} \pm q\right)^{2}+p_{ \pm}^{2}=k_{0}^{2}$. Как видно из (5), дифракция всегда соответствует процессам с переворотом спина. Подставляя (5) в (4), легко найти точные алгебраические выражения для неизвестных амплитуд волновых функций. Эти выражения громоздкие и в настоящей работе приводиться не будут. Коэффициенты прохождения по потоку вычисляются как отношение квадратов амплитуд волновых функций и проекций волновых векторов на ось $x$. Например, для дифракционных пиков справедливо выражение

$$
T_{+-,-+}=\frac{p_{+,-}\left|t_{12}, t_{21}\right|^{2}}{p_{0}\left(\left|i_{1}\right|^{2}+\left|i_{2}\right|^{2}\right)} .
$$

Обсудим некоторые свойства прошедшего пучка. Невзаимность рассеяния состоит в том, что интенсивность рассеяния неполяризованных нейтронов в некомпланарных магнитных полях изменяется, когда источник и детектор меняются местами [6]. С другой стороны, такая перемена мест источника и детектора эквивалентна смене направления магнитного поля в каждой точке на противоположное.

Рассмотрим процесс дифракции на геликоиде с переворотом спина из состояния „-“ в состояние „+ + “ при прохождении. Сменим направление магнитного поля на противоположное и воспользуемся симметрией дифференциального сечения рассеяния относительно вращений [6], а именно после инверсии магнитного поля повернем поле вокруг оси $z$ на угол $\pi$

$$
\begin{gathered}
T_{-+}\left(-B_{x},-B_{y},-B_{z}\right)=T_{-+}\left(\hat{R}_{z, \pi}\left(-B_{x},-B_{y},-B_{z}\right)\right) \\
=T_{-+}\left(B_{x}, B_{y},-B_{z}\right) .
\end{gathered}
$$

Иначе говоря, для измерения невзаимности при малоугловой дифракции на геликоиде достаточно только сменить направление внешнего магнитного поля, без изменения остальных проекций геликоидального поля.

\section{3. Обсуждение результатов}

Ядерный потенциал, величина намагниченности и характеристики геликоида взяты из работ $[8,12]$. В общем случае дифракция на решетке с волновым числом $q$ имеет резонанс для дифракционного пика порядка „-1“ в случае, когда проекция $k_{0 z} \approx q / 2$. Соответственно следует ожидать резонансного поведения и для пика $T_{-+}(\mathbf{B})$. Абсолютная величина внешнего поля составляет $1 \mathrm{kOe}$. В зависимости от направления приложенного поля меняется положение дифракционного пика. Покажем, что сдвиг дифракционного максимума возможен только в некомпланарной системе. Допустим, что кристалл $\mathrm{MnSi}$ с плоским геликоидальным распределением и внешнее поле представляют собой два независимых рассеивателя. Плоский геликоид является неколлинеарным и разрешает процессы с переворотом спина. Внешнее поле аналогично однородно намагниченному зеркалу, и соответственно преломление на нем зависит от того, направлен ли спин нейтрона по полю или против поля. Как показано в работе [6], невзаимные эффекты наблюдаются только за рамками однократного, борновского приближения. 


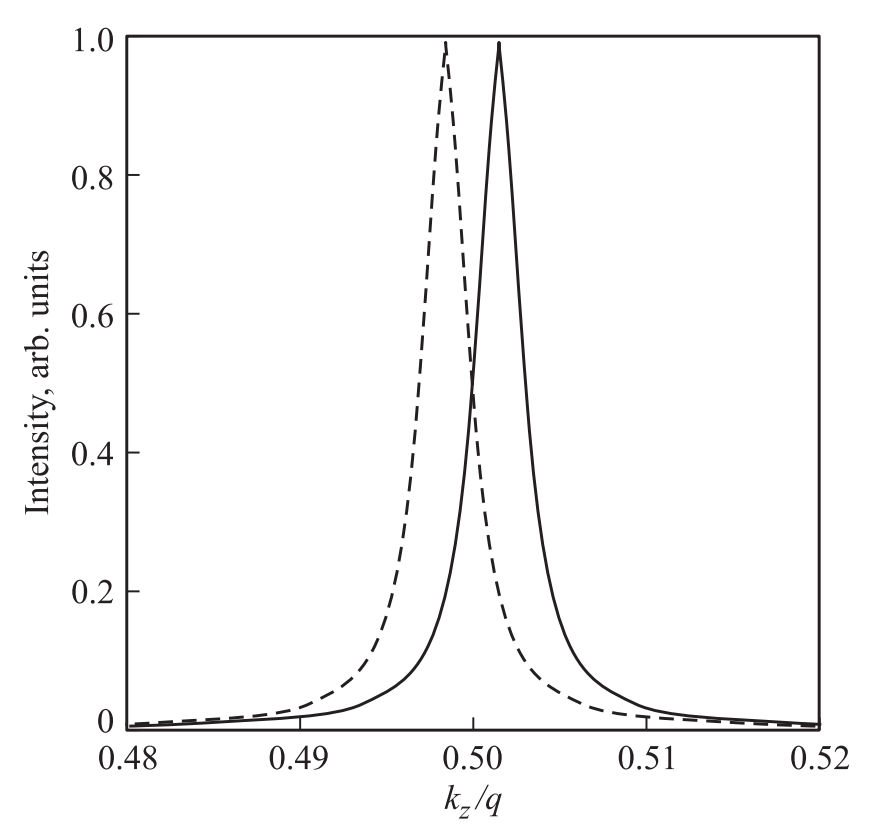

Рис. 2. Интенсивность дифракции при прохождении, соответствующая процессу с переворотом спина из состояния „-“ в состояние „+““. Сплошная кривая соответствует случаю, когда поле $B_{\text {ext }}$ приложено вдоль оси $z$, штриховая - случаю, когда поле приложено против оси $z$.

Для простоты рассмотрим процесс двукратного рассеяния. Тогда после дифракции на геликоиде с переворотом из состояния „-““ в состояние „,+“ пучок также преломляется и в однородном внешнем магнитном поле. Ясно, что в этом случае интенсивность прохождения зависит от взаимной ориентации спинового состояния „ + “ и внешнего поля. Учитывая резонансный характер $T_{-+}(\mathbf{B})$ вблизи $k_{0 z} \approx q / 2$, мы будем получать сдвиг пика резонанса, линейный по магнитному полю (рис. 2). Величина сдвига составляет порядка $0.01 q \approx 3 \cdot 10^{-4} \AA^{-1}$. Анализ характеристик спектрометров по малоугловому рассеянию нейтронов [13-15] показывает, что требуемое угловое разрешение достижимо.

\section{4. Заключение}

В работе представлены результаты точного аналитического расчета малоугловой дифракции нейтронов на нецентросимметричных кубических магнетиках с геликоидальным распределением намагниченности. Показано, что при приложении внешнего магнитного поля параллельно оси геликоида наблюдается сдвиг пика малоугловой дифракции при прохождении нейтронов. При этом от того, приложено магнитное поле вдоль оси геликоида или против, зависит и направление сдвига пика дифракции. Величина этого сдвига сравнима с точностью измерений на современных спектрометрах малоуглового рассеяния нейтронов.

\section{Список литературы}

[1] S.V. Grigoriev, A.S. Sukhanov, S.V. Maleyev. Phys. Rev. B 91, 224429 (2015).

[2] С.В. Григорьев, В.А. Дядькин, С.В. Малеев, D. Menzel, J. Schoenes, D. Lamago, E.B. Москвин, H. Eckerlebe. ФТT 52, 852 (2010).

[3] И.Е. Дзялошинский. ЖЭТФ 46, 1420 (1964).

[4] T. Moriya. Phys. Rev. 120, 91(1960).

[5] P.Bak, M.H.Jensen. J. Phys. C 13, L881 (1980).

[6] Д.А. Татарский, О.Г. Удалов, А.А. Фраерман. ЖЭТФ 142 , 710 (2013).

[7] O.G. Udalov. J. Phys. Soc. Jpn. 82, 064714 (2013).

[8] O.G. Udalov, A.A. Fraerman. Phys. Rev. B 90, 064202 (2014).

[9] Д.А. Татарский, А.В. Петренко, С.Н. Вдовичев, О.Г. Удалов, Ю.В. Никитенко, А.А. Фраерман. Письма в ЖЭТФ 102, 721 (2015).

[10] M. Calvo. Phys. Rev. B 18, 5073 (1978).

[11] О.Г. Удалов, А.А. Фраерман. ЖЭТФ 131, 71 (2007).

[12] C. Pappas, E. Lelièvre-Berna, P. Falus, P.M. Bentley, E. Moskvin, S. Grigoriev, P. Fouquet, B. Farago. Phys. Rev. Lett. 102, 197202 (2009).

[13] http://nrd.pnpi.spb.ru/wwrm/two1n.html

[14] http://flnp.jinr.ru/201/

[15] https://www.ill.eu/instruments-support/instruments-groups/ instruments/d22/characteristics/ 